\title{
Report on betatron phaseadvance measurements with phaseshifter in run 12
}

\author{
M. Bai, Y. Luo, A. Marusic, \\ M. Minty, G. Robert-Demolaize
}

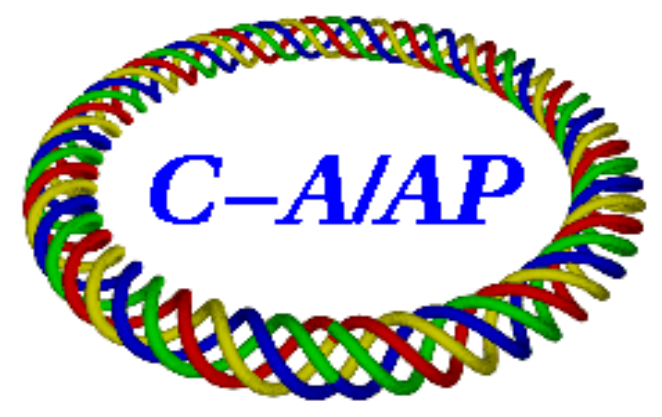

\section{Collider-Accelerator Department Brookhaven National Laboratory Upton, NY 11973}

Notice: This document has been authorized by employees of Brookhaven Science Associates, LLC under Contract No. DE-AC02-98CH10886 with the U.S. Department of Energy. The United States Government retains a nonexclusive, paid-up, irrevocable, world-wide license to publish or reproduce the published form of this document, or allow others to do so, for United States Government purposes. 


\title{
Report on Betatron Phaseadvance Measurements with Phaseshifter in RUN 12*
}

\author{
M. Bai, Y. Luo, A. Marusic, M. Minty, C. Montag, G. Robert-Demolaize \\ Brookhaven National Laboratory, Upton, NY 11973, U.S.A
}

The function of upcoming E-lens requests the phase advance between the collision IP and location of E-lens(IP10) to be integer multiples of pi. The RHIC quadrupole mains were modified(phaseshifter) to allow the control of phase advance between IP8 and IP10 [1]. Measurements were made during RHIC run 2012 to confirm the effect of phaseshifter on the betatron phase advance between the two IPs. The results are reported in this paper.

\section{INTRODUCTION}

Currently, RHIC polarized proton luminosity performance is limited by the tune shift induced by beam-beam. One electron lens is scheduled to be installed in each of the two rings to compensate the beam-beam tune shift [1]. In each ring, the electron lens will be located at IP10. In order to compensate the non-linear beam-beam tune shift, it is very critical to keep the phase advance between the Elens and the collision point to be integer multiples of $\pi$ [2]

Since RHIC was designed to have all the focusing/defocusing quadrupoles in the arcs to share the same power supply, two additional shunt power supplies, also known as phase shifter, were added to the arc quadrupoles in each ring to allow phase advance changes between IPs [1]. The phase shifters were finally integrated into the RHIC quench protection system during the RHIC run in 2012 by Don Bruno and his group. Fig. 1 and Fig. 2 show the calcuated phase advance change between the two IPs as a function of the phase shifter current. The effect of phase shifters on

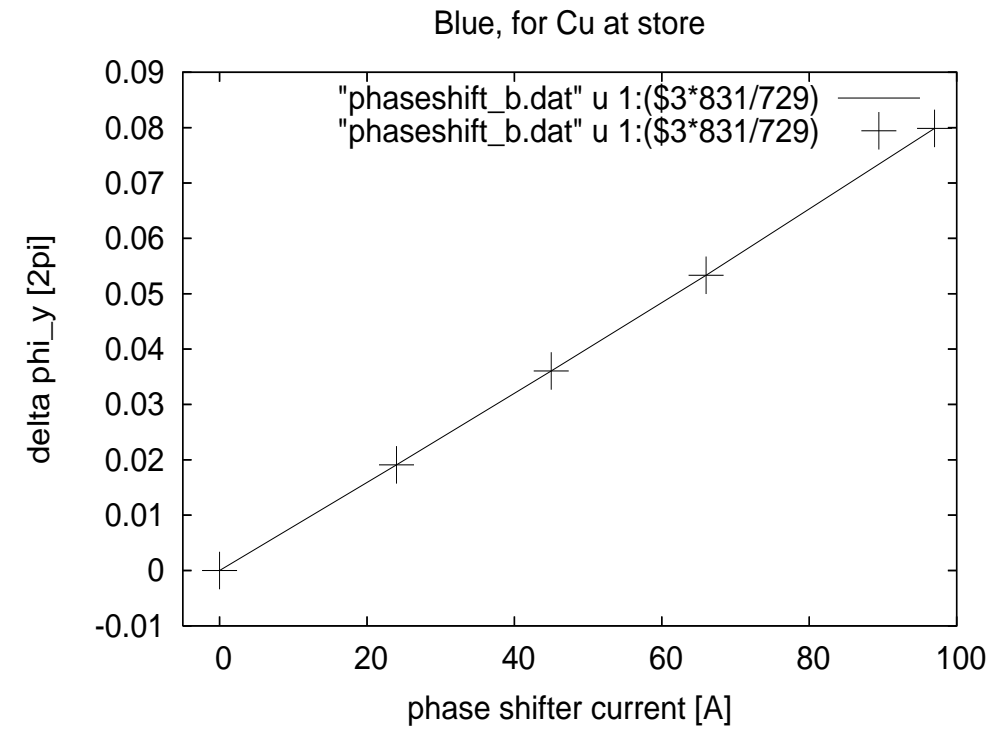

FIG. 1: This plot shows the calculated phase advance change between IP8 and IP10 as function of phase shifter current in the Blue ring.

beam optics was also measured during the run.

${ }^{*}$ The work was performed under the auspices of the US Department of Energy. 


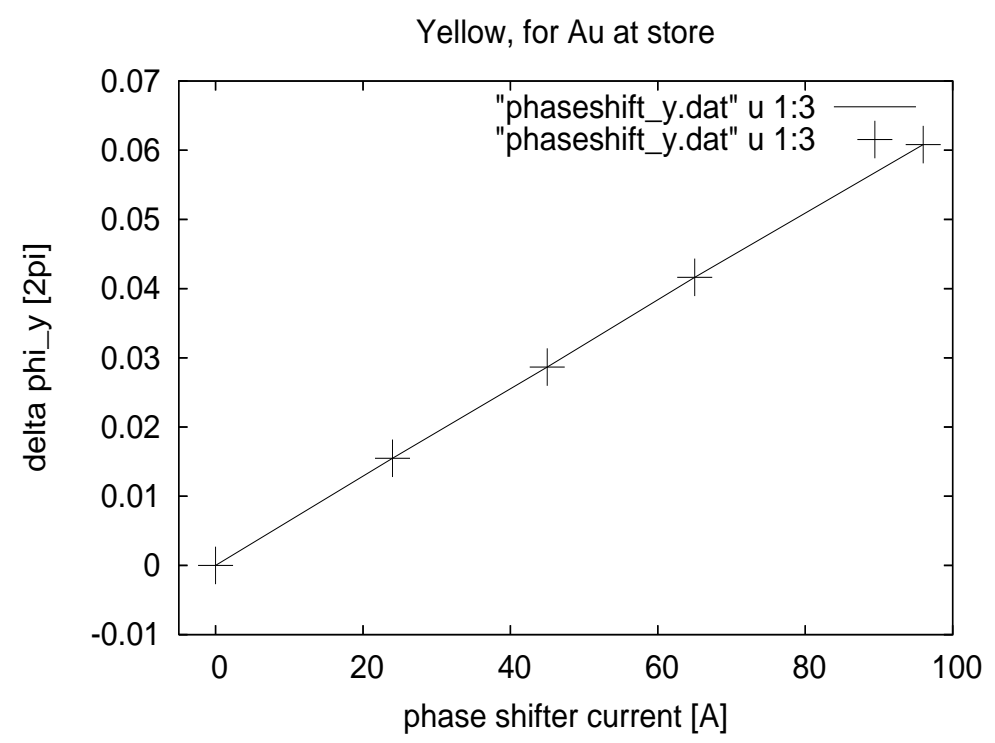

FIG. 2: This plot shows the calculated phase advance change between IP8 and IP10 as function of phase shifter current in the Yellow ring.

\section{OPTICS MEASUREMENT USING AN AC DIPOLE}

The technique of using an ac dipole with an oscillating magnetic field to excite a coherent betatron oscillation was first tested and applied in the Brookhaven AGS [3]. Since then, it has been adopted by RHIC and by the Tevatron and will also be installed in the LHC [4-7]. A sizable long lasting coherence can be induced without blowing up the beam emittance when the driven coherence is excited adiabatically. This makes it more attractive for hadron accelerators to conduct optics measurements, and beam dynamics studies.

Without losing generality, assume a single ac dipole with vertical magnetic field in a linear accelerator. At every orbital revolution when a particle passes by the ac dipole, it gets an horizontal deflection $\Delta x_{p}^{a c}(k)$ given by

$$
\Delta x_{p}^{a c}(k)=\frac{B_{y}^{a c} L}{B \rho} \beta_{x}^{a c} \cos \left(2 \pi Q_{x}^{a c} k+\chi_{x}^{a c}\right)=\frac{B_{y}^{a c} L}{2 B \rho} \beta_{x}^{a c}\left(e^{i\left(2 \pi Q_{x}^{a c} k+\chi_{x}^{a c}\right)}+e^{-i\left(2 \pi Q_{x}^{a c} k+\chi_{x}^{a c}\right)}\right)
$$

where $k$ is the turn number, $B \rho$ is the magnetic rigidity, $\beta_{x}^{a c}$ is the horizontal beta function at the location of the ac dipole, $B_{y}^{a c} L$ is the amplitude of the ac dipole integrated oscillating field strength, $Q_{x}^{a c}$ is the ac dipole driving tune (the ac dipole field oscillating frequency divided by the revolution frequency) and $\chi_{x}^{a c}$ is the ac dipole oscillating field initial phase. Let

$$
z_{x}^{a c}=x^{a c}+i x_{p}^{a c} .
$$

Here, $x^{a c}$ and $x_{p}^{a c}$ are the normalized coordinates at the location of the ac dipole. Since the transfer map from the exit of the ac dipole back to the entrance of the ac dipole in one turn is $e^{i 2 \pi Q_{x}}$, the normalized coordinates between a two successive turns are related by

$$
z_{x}^{a c}(k+1)=e^{i 2 \pi Q_{x} k}\left(z_{x}^{a c}(k)+\Delta x_{p}^{a c}(k)\right) .
$$

Here, $Q_{x}$ is the beam horizontal betatron tune. The solution of Eq. 3 in the condition of adiabatically ramping the ac dipole oscillating field from zero to $B_{y}^{a c} L$ yields the exact solution of the driven betatron oscillation as shown in Eq. 4.

$$
z_{x}^{a c}(k)=A_{m}^{a c} e^{i\left(2 \pi Q_{x}^{a c} k-\pi\left(Q_{x}^{a c}-Q_{x}\right)+\chi_{x}^{a c}\right)}-A_{p}^{a c} e^{-i\left(2 \pi Q_{x}^{a c} k-\pi\left(Q_{x}^{a c}+Q_{x}\right)+\chi_{x}^{a c}\right)} .
$$

with

$$
A_{m}^{a c}=\frac{B_{y}^{a c} L}{4 \sin \left(\pi\left(Q_{x}^{a c}-Q_{x}\right)\right) B \rho} \beta_{x}^{a c}
$$


and

$$
A_{p}^{a c}=\frac{B_{y}^{a c} L}{4 \sin \left(\pi\left(Q_{x}^{a c}+Q_{x}\right)\right) B \rho} \beta_{x}^{a c}
$$

Here, one also assumes that the initial betatron oscillation is negligible. Detailed derivation can be found in the RHIC/AP/159 [8]. The normalized coordinates $x^{a c}(k)$ and $x_{p}^{a c}(k)$ at the ac dipole are then

$$
\begin{array}{r}
x^{a c}(k)=\frac{B_{y}^{a c} L}{4 \sin \left(\pi\left(\mathrm{Q}_{\mathrm{x}}^{\mathrm{ac}}-\mathrm{Q}_{\mathrm{x}}\right)\right) \mathrm{B} \rho} \beta_{x}^{a c} \cos \left(2 \pi Q_{x}^{a c} k-\pi\left(Q_{x}^{a c}-Q_{x}\right)+\chi_{x}^{a c}\right)- \\
\frac{B_{y}^{a c} L}{4 \sin \left(\pi\left(\mathrm{Q}_{\mathrm{x}}^{\mathrm{ac}}+\mathrm{Q}_{\mathrm{x}}\right)\right) \mathrm{B} \rho} \beta_{x}^{a c} \cos \left(2 \pi Q_{x}^{a c} k-\pi\left(Q_{x}^{a c}+Q_{x}\right)+\chi_{x}^{a c}\right) .
\end{array}
$$

and

$$
\begin{array}{r}
x_{p}^{a c}(k)=\frac{B_{y}^{a c} L}{4 \sin \left(\pi\left(Q_{x}^{a c}-Q_{x}\right)\right) B \rho} \beta_{x}^{a c} \sin \left(2 \pi Q_{x}^{a c} k-\pi\left(Q_{x}^{a c}-Q_{x}\right)+\chi_{x}^{a c}\right)+ \\
\frac{B_{y}^{a c} L}{4 \sin \left(\pi\left(Q_{x}^{a c}+Q_{x}\right)\right) B \rho} \beta_{x}^{a c} \sin \left(2 \pi Q_{x}^{a c} k-\pi\left(Q_{x}^{a c}+Q_{x}\right)+\chi_{x}^{a c}\right) .
\end{array}
$$

For any other location in the machine, the normalized betatron coordinates are obtained through the rotation matrix between the ac dipole and the observation location $s$

$$
\left(\begin{array}{l}
x^{s} \\
x_{p}^{s}
\end{array}\right)=\sqrt{\frac{\beta_{x}^{s}}{\beta_{x}^{a c}}}\left(\begin{array}{cc}
\cos \Delta \psi_{s} & \sin \Delta \psi_{s} \\
-\sin \Delta \psi_{s} & \cos \Delta \psi_{s}
\end{array}\right)\left(\begin{array}{l}
x^{a c} \\
x_{p}^{a c}
\end{array}\right)
$$

which yields

$$
x^{s}(k)=A_{m} \cos \left(2 \pi Q_{x}^{a c} k-\pi\left(Q_{x}^{a c}-Q_{x}\right)+\chi_{x}^{a c}-\Delta \psi_{s}\right)-A_{p} \cos \left(2 \pi Q_{x}^{a c} k-\pi\left(Q_{x}^{a c}+Q_{x}\right)+\chi_{x}^{a c}+\Delta \psi_{s}\right)
$$

with

$$
A_{m}=\frac{B_{y}^{a c} L}{4 \sin \left(\pi\left(Q_{x}^{a c}-Q_{x}\right)\right) B \rho} \sqrt{\beta_{x}^{a c} \beta_{x}^{s}}
$$

and

$$
A_{p}=\frac{B_{y}^{a c} L}{4 \sin \left(\pi\left(Q_{x}^{a c}+Q_{x}\right)\right) B \rho} \sqrt{\beta_{x}^{a c} \beta_{x}^{s}} .
$$

Here, $\beta_{x}^{s}$ is the beta function at location $s . \Delta \psi_{s}$ is the phase advance between the ac dipole and location $s$. Evidently, the driven oscillation consists of two terms which have the same driving tunes but with different phases. Since in general one places the ac dipole driving tune very close to the betatron tune, the driven oscillation is dominated by the term with the amplitude $A_{m}$. However, the term with the amplitude $A_{p}$ can be non-negligible if the betatron tune is close to either an integer or a half integer.

\section{MEASURED PHASE ADVANCE IN BLUE}

The phase advance at each BPM in the Blue ring was measured with $\mathrm{Cu}$ beam at store energy with different phase shifter currents. Fig. 3 shows the measured phase advance around the ring for phase shifter current at 10 A and 100 A. Fig. 4 shows the difference of the measured phase advance at all available beam position monitors (bpms) with phase shifter at 100 Amp and at 10 Amp. The measurement shows that a total of 32 degrees vertical phase advance was introduced by phase shifter, while the accumulated phase advance over the ring remained the same. The measurement was caried out with $\mathrm{Cu}$ beam at its top energy with tune and coupling feedback on to keep overall betatron tune stay fixed. 

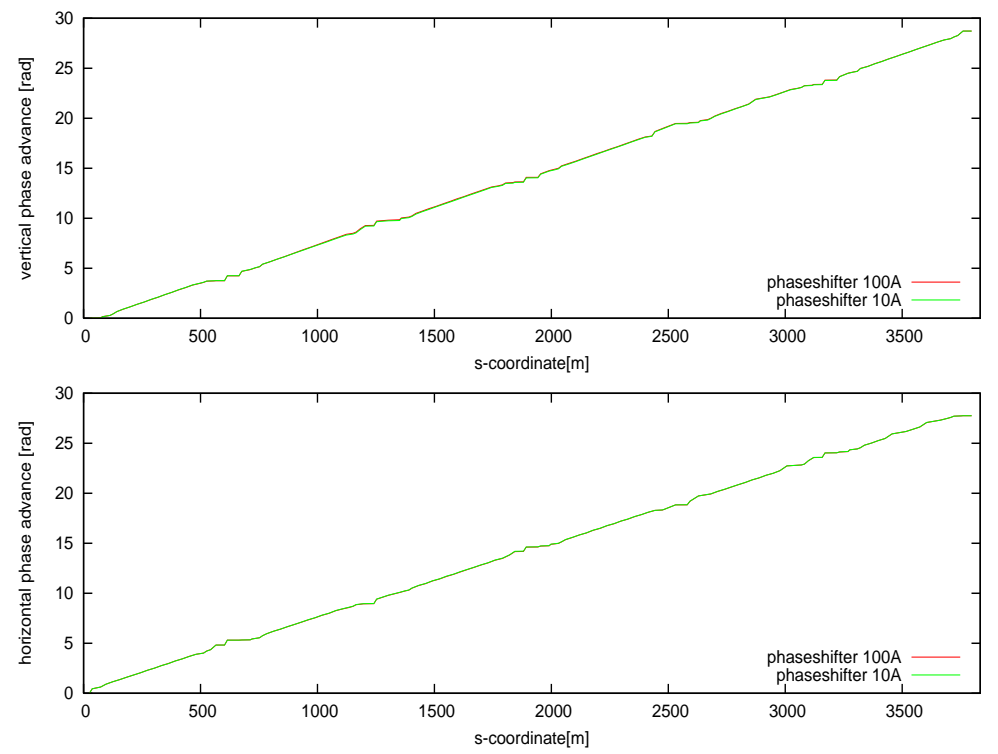

FIG. 3: The top plot shows the measured vertical phase advance around the ring and the bottom plot shows the measured horizontal phase advance for each bpm. Red line corresponds to the phase shifter at $100 \mathrm{~A}$, and green line is for phase shifter at $10 \mathrm{~A}$.
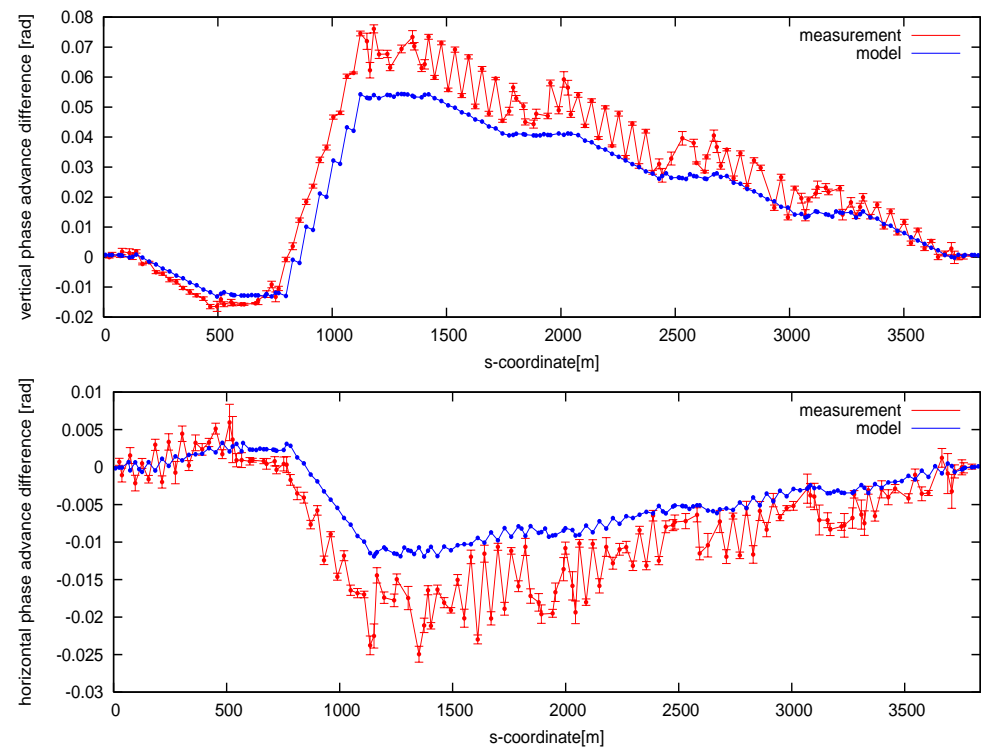

FIG. 4: The top plot shows the measured vertical phase advance difference between phase shifter current at $100 \mathrm{~A}$ and phase shifter current at $10 \mathrm{~A}$. The bottom plot shows the measured horizontal phase advance between the two phase shifter settings.

\section{MEASURED PHASE ADVANCE IN YELLOW}

The phase advance at each BPM in the Yellow ring was measured with $\mathrm{Cu}$ beam at store energy with different phase shifter currents. Fig. 3 shows the measured phase advance around the ring for phase shifter current at $10 \mathrm{~A}$ and 100 A. Fig. 6 shows the difference of the measured phase advance at all available beam position monitors (bpms) with phase shifter at $100 \mathrm{Amp}$ and at $10 \mathrm{Amp}$. The measurement shows that a total of 32 degrees vertical phase advance was introduced by phase shifter, while the accumulated phase advance over the ring remained the same. The measurement was caried out with Au beam at its top energy with tune and coupling feedback on to keep overall betatron tune stay fixed. 

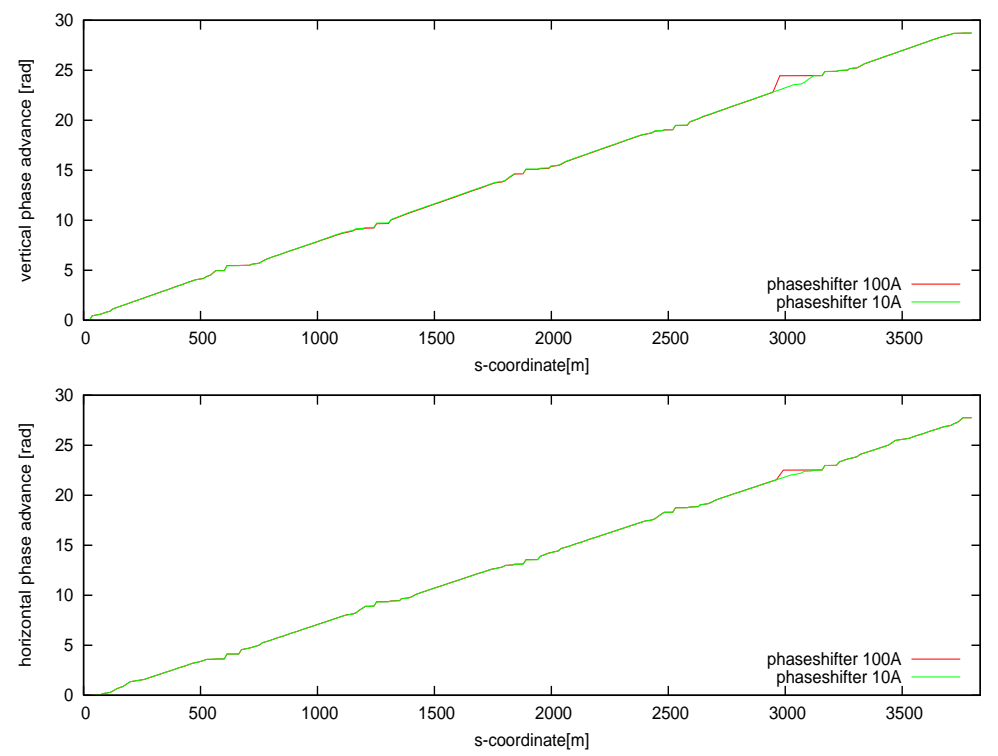

FIG. 5: The top plot shows the measured vertical phase advance around the ring and the bottom plot shows the measured horizontal phase advance for each bpm. Red line corresponds to the phase shifter at $100 \mathrm{~A}$, and green line is for phase shifter at $10 \mathrm{~A}$.
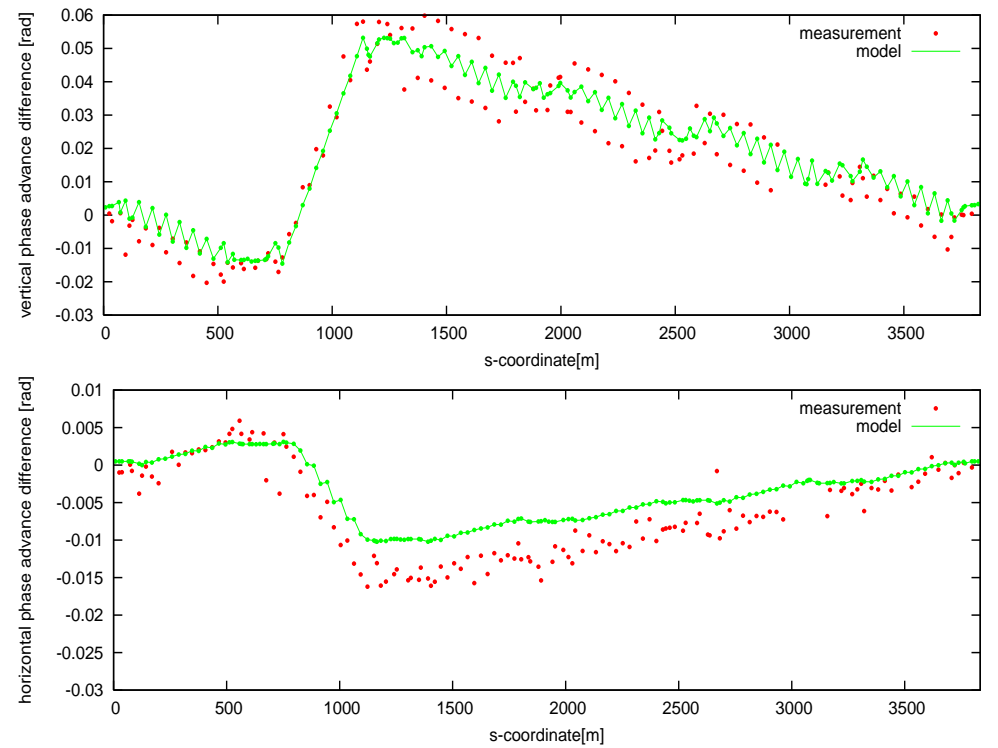

FIG. 6: The top plot shows the measured vertical phase advance difference between phase shifter current at $100 \mathrm{~A}$ and phase shifter current at $10 \mathrm{~A}$. The bottom plot shows the measured horizontal phase advance between the two phase shifter settings.

\section{CONCLUSION}

The phase adance was measured with different phase shifter current was measured in both Blue and Yellow. The measurement of phase change due to phase shifter agrees well with model calculation for both cases.

\section{ACKNOWLEDGMENT}

The author would like to thank P. Thieberg for his independent data analysis using least square fitting. which helped greatly to discover the arbitrary phase error of $2 \pi$ at some bpms. This is due to the fact that it is not possible 
to determine integer part of the phase at each bpm by fitting its turn by turn data.

[1] W. Fischer, Y. Luo, A. Pikin, RHIC electron lenses design report, BNL C-A/AP/359. 2010

[2] Y. Luo et al Six-dimensional weak-strong simulation of head-on beam-beam compensation in the Relativistic Heavy Ion Collider, PRST-AB 15, 051004, 2012

[3] M. Bai et al Experimental test of coherence betatron excitations, Phys. Rev. E56, 6002, (1997)

[4] M. Bai et al RHIC AC Dipole Design and Construction, Proceedings of PAC01, 3606, Chicago, USA (2001)

[5] R. Miyamoto et al, Proceedings of PAC07, 3868, Albuquerque, New Mexico, USA (2007)

[6] O. Berrig et al Excitation of Large Transverse Beam Oscillations without Emittance Blow-up Using the AC-Dipole Principle, Proceedings of DIPAC 2001, 82, Grenoble, France (2001)

[7] J. -P. Koutchouk Advanced Diagnostics of Lattice Parameters in Hadron Colliders, Proceedings of DIPAC 2003, 45, Mainz, Germany (2003)

[8] S. Peggs and C. Tang Nonlinear Diagnostics Using an AC Dipole, RHIC/AP Report 159, (1998)

[9] R. Miyamoto et al, Proceedings of PAC07, 3465, Albuquerque, New Mexico, USA (2007) 